\title{
VIII.
}

\section{Die Philosophie in Dänemark im 19. Jahrhundert.}

\section{Von \\ Professor Harald Höffding in Kopenhagen.}

Das geistige Leben hier im Norden hat sich - seit der Reformationszeit - in genauem Zusammenhange mit und unter starkem Einflusse von dem deutschen Geistesleben entwickelt. Dies gilt besonders in philosophischer Rücksicht. Aus Dänemark ist kein Denker hervorgegangen, welcher einen Wendepunkt in der Geschichte des philosophischen Forschens herbeigeführt hätte. Aber wie auf anderen Gebieten hat man auch hierin die vom Auslande kommenden Strömungen auf eigentümliche Weise zu assimiliren und zu verarbeiten versucht. Im Anfange dieses Jahrhunderts trat bei uns die Philosophie der Romantik auf und übte einen grossen, Anfangs sogar einen überwältigenden Einfluss auf unser ganzes Geistesleben, besonders auf die Poesie und die Entwickelung der religiösen Ideen aus. Unsere neuere nationale Literatur wurde durch diese Einwirkung und die dadurch erweckte frische und tiefe Lebensauffassung hervorgerufen. Die Philosophie Schellings und Hegels ïbte lange Zeit eine grosse Herrschaft. Aber man darf behaupten, dass der der dänischen Geistesrichtung eigentümliche Sinn fïr die reale Natur und die psychologische wie geschichtliche Virklichkeit es uns möglich machte, dass die bedeutungsvollen Ideen jener Philosophie unsere Entwicklung befruchten konnten, ohne dass wir uns doch in abstrakte und hyperidealistische Speculationen verloren. - In den letzten Decennien hat sich bei uns wie in Deutschland dic kritische Philosophie wieder hervorgearlseitet. Neben

A rohit f. Gesthithte a. Pliblusophite. II. 
deutscher Einwirkung geht jetzt ein sehr starker Einfluss von England aus, indem die jüngere Generation von Stuart Mill, Darwin und Herbert Spencer entscheidende Impulse empfangen hat.

I.

Henrik Steffens, in Norwegen (1773) geboren, auf väterlicher Seite aus Holstein, auf mütterlicher aus einem altdänischen, bis ins Mittelalter zurückgehenden Geschlechte stammend, ein feuriger. Redner, welcher besonders die Gabe besass die Eigentümlichkeit anderer $\mathrm{zu}$ erblicken und $\mathrm{zu}$ erwecken, führte die Schellingsche Philosophie in Dänemark durch die in der dänischen Litteraturgeschichte berühmten Vorlesungen ein, welche er im November 1802 in Kopenhagen begann, und welche im folgenden Jahre teilweise gedruckt wurden. (Indledning til filosofiske Foreloesninger 1803). Er stellte hier die nąturphilosophischen Gedauken dar, welche er früher in einer deutschen Schrift (Beiträge zur innern Naturgeschichte der Erde, 1801) ausführlich entwickelt hatte; aber er behandelte auch Probleme, welche der Aesthetik, der Religionsphilosophie und der Philosophie der Geschichte angehörten. Es war ja eben die Aufgabe der Schellingschen Philosophie, den inneren $\mathrm{Zu}$ sammenhang oder gar die Identität aller dieser Probleme zu zeigen. Sie machte keinen scharfen Unterschied zwischen Geist und Natur, zwischen dem Bewussten und dem Unbewussten, zwischen dem Gedanken und der Phantasie, zwischen Gott und dem Universum. Die Natur ist eine Stufenreihe, innerhalb welcher das geistige Leben sich nach und nach entwickelt. In allem ist eine und dieselbe Einheit; in der Welt der Natur und der des Geistes regen sich dieselben Kräfte und dasselbe Leben, wenn das Auge nur aufgetan ist sie zu erblicken. Steffens begann darum seine Vorlesung mit der. Erklärung, dass er bei seinen Zuhörern „den inneren Drang, das eigentliche Wesen der Dinge zu erkennen, das Rätsel des Daseins zu lösen, und die innere Anschauung, welche jeden Teil zu einem Ganzen zusammenfasst und das absolute Ganze und Eine àls das Reale setzt", voraussetzte. Er appellirte also an ein höheres Organ, eine „intellektuelle Anschauung“. Er suchte dann die Einheit in der Natur und in der Geschichte aufzuzeigen. Es gilt für 
ihn „die Freiheit der Vernunft in der Notwendigkeit der Natur, die Notwendigkeit der Natur in der Geschichte zu erkennen". Die unorganische Welt erreicht erst in der organischen ihr eigenes Ziel, und innerhalb dieser entsteht der freie Geist, dessen notwendige Entwickelung die Geschichte darbietet. Durch die ganze Natur ist eine individuálisirende Kraft zu spüren, die im Menschen ihre Vollendung erreicht. In der poetischen Genialität und der religiösen Begeisterung haben wir die höchsten Offenbarungsformen der grossen, in Allem sich regenden Kraft vor uns. Aber dies alles ist nur einer höheren Anschaunng zugänglich, während es dem experimentellen Forschen und dem kritischen Verstande verborgen ist. "Uusere Wissenschaften haben uns die Natur verschlossen!“ Von den physischen und chemischen Theorien appellirt Steffens kühn an "die ewige Natur selbst". Er wollte einen Standpunkt einnehmen, auf welchem sich die höchste Wissenschaft, die höchste Religiosität und die höchste Poesie begegneten, und legte einen gewaltigen Protest gegen die kritische, analysirende Tendenz des 18. Jahrhunderts ein. Als ächter Romantiker pries er die Ur\%eit, als das Geistesleben noch ungeteilt war, und die Menschen der Natur und der Gottheit näher standen. „Ist nicht das Schönste, Herrlichste, was unsere Zeit durchdringt, der Sinn für jene alte, rerschwundene?" - Diesen Vorlesungen verdanken nach ihren eigenen Erklärungen die hervorragendsten Führer der poetischen und religiösen Entwicklung in Dänemark, Männer wie Oehlenschläger, Grundtrig und Mynster, ihre erste Anregung. Vielerlei Ideen wurden angeregt; ein reicher Gährungsstoff war jetzt vorhanden. Steffens selbst mar es nicht vergönnt, diese Keime zu pflegen; die Autoritäten betrachteten ihn mit Nisstrauen; man beschuldigte ihn, bald dem Atheismus, bald dem Katholicismus zu huldigen. Er war auch mehr dazu geeignet die Bewegung hervorzurufen als sie zu leiten. 1804 ging er nach Halle, und seine weitere Entwicklung gehört der deutschen Literatur an.

Einem klareren und besonneneren Denker war es vorbehalten, das philosophische Forschen hier in J)änemark wciter zu führen. Im Jahre 1803 wurde Xiels Treschow (geb. bei J)rammen in Norwegen 1751, gest. in Kristiania 1893), welcher als Rektor in Kristiania cine 

gibt kein Kausalverhältniss zwischen Empfindung und Bewegung; Bewegung kann nur Bewegung, Empfindang nur Empfindung hervorbringen. Aber jede Bewegung ist mit einer entsprechenden Empfindung, jede Empfindung mit einer Bewegung verbunden. Die allgemeine Materie ist der, sichtbare Gott, eine Offenbarung des höchsten Wesens; die Kräfte der Natur sind von der Kraft der Gottheit nicht verschieden. - Die Klarheit, mit welcher Treschow die Identitätshypothese aufstellt, die seine Lehre vor der SchellingHegelschen auszeichnet, wird doch durch seinen Vitalismus einigermassen gestört. Die Seele ist nach ihm mit der "Lebenskraft" eins, und wirkt als solche anch in den körperlichen Funktionen. Dieser mythologische Begriff, welchen er mit der Physiologie seiner Zeit teilt, macht es ihm nur anscheinend leichter das Problem zu lösen.

Durch die ganze Natur und Geschichte nimmt Treschow eine kontinuirliche Entwickelung an. „Von Stoffen, welche roh $z u$ sein scheinen, weil man noch keine Form in ihnen entdeckt, vollzieht sich ein allmählicher Uebergang zu bestimmteren Gestalten, deren Reihenfolge nur in allgemeinen Zügen angegeben werden kann. Durch scharf bezeichnete Grenzen sind sie nicht zu unterscheiden, weil der Gang der Natur in den Abweichungen oder Ausartungen, durch welche die früheren Geschlechter und Arten in ganz neue verwandelt werden, nicht blos kriechend ist, sondern so langsam, dass weder Erfahrung noch Geschichte uns befähigen, seinen beinahe unmerklichen Spuren $z$ folgen. "Alles Lebendige hat dieselbe $W^{\top}$ urzel. Wemn wir hinlänglich zurückgehen, kommen wir zu einer Stufe, "wo das moralische Wesen kaum vom physischen zu unterscheiden war, - wo der Mensch noch nicht Mensch war". Nun zeigt uns die Geologie, dass es Wasserthiere gab, noch ehe das feste Land seine Einwohner bekam. Auch der Mensch muss also Wasserthiere unter seinen Ahnen haben. Wir haben uns dieses Ursprungs nicht zu schämen. Ist ja doch jedes Individuum noch immer in seinem Anfang ein Wesen, das tief unter vielen Thieren, ja Pflanzen steht. Die Geschichte des Individuums und des Geschlechts bietet dieselben Grundzüge. - Treschow bewundert also nicht, wie die Romantikes, die Urzeit. Für ihn 
streitet es gegen die allgemeinen (iesetze der Entwickelung, dass die ersten Menschen Genien oder Götter gewesen sein sollten, und nicht Instinktwesen, welche erst durch einen langen und mühsamen Entwickelungsgang Vernunftwesen werden konnten. Er nimmt bei allen lebendigen Wesen einen Entwickelungstrieb an, welcher besonders als ein Trieb nach Selbstwirksamkeit hervortritt. Im Menschen geht dieser Trieb endlich darauf aus, nach Vernunftideen selbstwirksam $\%$ sein. - Diese Lehre stärkt nach 'Treschow unsere Hoffnung und unsere Geduld. Sie macht die Nenschheit gross und heilig in unseren Augen, indem wir sehen, dass an ihrer Vollkommenheit seit Jahrtausenden gearbeitet wurde und noch immer gearbeitet wird.

In seiner Schrift "Ueber die menschliche Natur, besonders von der geistigen Seite" gibt Treschow eine klare und gesunde Darstellung der psychologischen Hauptphänomene und Gesetze. Sie zerfällt in drei Abschnitte: 1. Ueber das Vorstellungsvermögen (hierunter: über die Empfindungen, über die Reproduktion und die Association, ïber die Denkkraft). 2. Ueber das Gefühlsvermögen. 3. Ueber den Willen. (Hierunter auch ïber Triebe und Neigungen, über Gewohnheiten und Fertigkeiten, über Leidenschaften und Affekte.) - Obgleich Treschow nicht wenig von der vorkantischen Auffassung beeinflusst ist, weist er doch entschieden die Lehre Leibniz's von den Gefühlen als verworrenen Vorstellungen ab. Diese Lehre gründet sich, wie er zu zeigen sucht, auf eine unvollständige Induktion. - Im Einzelnen finden sich bei Treschow sehr viele feine und treffende Bemerkungen und Analysen. Von älteren Autoren scheint ihn besonders Tetens (welcher ja ein geborener Däne war) beeinflusst zu haben.

Die spätere Wirksamkeit Treschow's, nach seiner Rückkehr nach Kristiania, war besonders der Religionsphilosophie gewidmet. Er gab eine platonisirende "Christenthumsphilosophie" heraus. Aber seine Wirksamkeit in Kopenhagen hatte doch die Bedeutung, dass die Philosophie bei uns von dem Evangelium der Romantik sich entfernte, und dass naturwissenschaftliche, psychologische und historische Studien als Grundlagen der Philosophie behauptet wurden. 
Auch von der religiösen Seite her erhob man der romantischen Schule gegenüber ernste Bedenken. Unter den von Steffens beeinflussten Männern habe ich oben auch N. F. S. Grundtrig genannt, den grossen Dichter und eifrigen Vorkämpfer für Orthodoxie, Nationalität und volkstümliche Elziehung, dem unser ganzes Volksleben ausserordentlich viel verdankt. Philosophisches Studium und Denken hat in die Entwickelung dieses mächtigen Geistes stark hineingegriffen. Es gab eine Zeit, wo er, seiner eigenen Aussage nach, "mit Fichte, Schiller und Schelling über die Rätsel des Lebens sann". Besonders hat Fichte einen grossen Einfluss auf ihn gehabt, einen grösseren vielleicht, als er sich klar bewusst war, obgleich er ihn immer hoch verehrte und ihm, selbst nachdem er seinerseits der weltlichen Wissenschaft den Rücken gekehrt hatte, durch ein Gedicht ein schönes Denkmal nach seinem Tode setzte. Nicht nur wirkten die ideale Begeisterung und der ethische Ernst Fichte's tief auf Grundtrig ein, sondern viele der historischen und pädagogischen Ideen des deutschen Philosophen (besonders diejenigen, welche er in den „Grundzügen des gegenwärtigen Zeitalters“ und den „Reden an die deutsche Nation“ entwickelte) findet man in den Anschaunngen des dänischen Dichtertheologen wieder. Der Kampf gegen die geistlose Gelehrsamkeit und das blosse Aufsammeln von Stoff, die Polemik gegen den allzu grossen Raum, den in neuerer Zeit das geschriebene ("todte") Wort auf Kosten des mündlichen ("lebendigen") eiunimmt, die Arbeit für eine nationale und volkstümliche Erziehung, welche die ursprünglichen Vermögen des Individuums und des Volks sich frei entfalten lässt und das Volk nicht in verschiedene Klassen zerspaltet, - alles dies kann Punkt für Punkt in Fichtes Schriften nachgrewiesen werden und gibt ein lehrreiches Beispiel davon ab, wie Gedanken in einem ganz andern Boden als demjenigen, in welchem sie zuerst hervorgesprossen sind, wurzeln und Früchte tragen können. Cnsere vorwiegend "nationale" Richtung in Schule und Leben verlankt der deutschen Philosophie einige ihrer bedeutendsten Ideen. Auch von Schelling wurde Grundtrig stark beeinflusst. DieSchellingsche Philosophie zeigte ihm die ganze Natur und die Geschichte als ein grosses Weltdrama, welches die Poesie, die Religion und die Wissen- 
schaft, jecle auf ibre Weise, daryustellen suchen. Als aber die Zeit fiir Crrundtrig kam, da sein gährender Geist in dem „alten, einfailtigen Glauben" Ruhe fand, musste er an einer Lehre Anstoss nehmen, nach welcher Gott nicht ist, sondern sich ewig durch das Woltleben ontwickelt; und fïr welche das christliche Dogma von der leidenden Gottheit, welche die Sünde durch ihren Tod sühnt, nur ein grosses Symbol der durch Leiden und Kämpfe fortschreitenden Weltentwicklung ist. Er schrieb dann (1815), als Antwort auf einen Angriff des Physikers H. B. Oersted, eine Streitschrift, in der el beweisen wollte, "daiss Schelling's Philosophie unchristlich, gottlos und lügenhaft ist". - An die Philosophie der Romantik erinnert doch auch später die Stellung Grundtrig's und vieler seiner Anhänger der experimentellen und kritischen Wissenschaft gegenüber. Er gab eigentlich niemals die Ansicht auf, welche ihn in der genannten Schrift aussprechen liess, dass "das leidenschaftliche Studium und die hohe Werthschätzung der Physik, besonders der experimentellen, auf Mechanik gegründeten, ein Zeichen des nahen Todes der Wissenschaftlichkeit ist". Sein Begriff von Wissenschaft trug, wie seine ganze Lebensanschauung, fortwährend das Gepräge der Romantik. Die geistigen Visionen mit ihrer Genialität und ihrem Irrtum waren bei ihm immer stärker als die prïfende Kritik. Der Sinn für die ideale Bedeutung der Erscheinungen wurde nicht mit Interesse für ihren realen Zusammenhang vereint. Hierin liegt die Begrenzung des an und für sich fruchtbaren Einflusses des grossen Mannes auf das Geistesleben unseres Volkes, eine Begrenzung, welche zum Teil der Zeit zu verdanken ist, innerhalb welcher seine Lehrjabre fielen. -

Der Gegner Grundtrig's, Hans Christian Oersted, der berühmte Physiker, Entdecker des Elektromagnetismus, war zuerst in seinẹr allgemeinen Naturauffassung ein Schüler Schelling's. Selbst später, als er mit nüchternen Augen auf die spekulative Naturphilosophie zurücksah, behauptete er, dass „die grosse Bewegung, welche geistreiche Männer am Anfang des Jahrhunderts in Rücksicht auf die wissenschaftliche Auffassung hervorgerufen hatten, einen wichtigen Einfluss auf die Naturforscher Deutschlands und des Nordens gehabt hat und auch nicht ohne Einfluss auf den wissen- 
schaftlichen Geist in der übrigen Welt geblieben ist". Die Idee der Einheit der Naturkräfte, welche die Schellingsche Philosophie behauptete, hat gewiss auch bei den Untersuchungen mitgewirkt, welche zu seiner berühmten Entdeckung Anlass gaben. Er sonderte später bestimmt zwischen Spekulation und Erfahrung und erklärte, dass es eben für den, welcher die Ueber\%eugung hat, dass eine Grundeinheit die ganze Natur durchdringt, doppelt notwendig wird, seinen Blick auf die Welt des Mannigfaltigen zu richten, innerhalb welcher diese Wahrheit erst seine Bestätigung finden kann. Und er meinte, dass sich eben aus einer gründlichen Naturforschung eine tiefere Philosophie entwickeln wïrde, als diejenige ist, welche sich in den meisten philosophischen Systemen findet, und eine echtere Poesie als diejenige, welche sich in den meisten Dichtungen findet, ja dass auch die religiösen Anschauungen dadurch berichtigt werden könnten.

In "Aanden i Naturen" (1849-50), eins der bedeutendsten Werke unserer Literatur (welches auch in deutscher Uebersetzung: "Der Geist in der Natur" vorliegt), sammelte Oersted eine Reihe ron Abhandlungen, welche zu sehr verschiedenen Zeiten verfasst sind, aber vereint ein klares und schönes Bild seiner Weltanschauung geben. Sein Hauptgedanke ist, dass dasjenige in der Natur, welches sich bei der ununterbrochenen Veränderung erhält, die Kräfte sind, welche zuletzt zu einer Grundkraft zurückführen, und die Gesetze, welche sich zuletzt als eine die ganze Natur durchdringende Allvernunft zeigen. Die körperliche Wirklichkeit ist nicht die wahre; die Körper sind nur Aeusserungen lebendiger Wirksamkeiten. Körper und Geist sind unzertrennlich in einem Princip rerbunden. Im Denken erwacht die schaffende Natur zum Bewusstsein in uns; daher sind wir fähig, die Natur zu verstehen. Zwischen Gottes Willen, der nicht als dem menschlichen ähnlich gedacht werden muss, und dem W'esen der Natur, kann kein Streit sein, weil beide eins sind. -

Auch auf specielleren Gebieten hat Oersted interessante Gesichtspunkte entwickelt. So z. B. in seinen „To Kapiter af deh Skönnes Naturlaere" (1845). (Anch in deutscher Uebersetzung: Naturlehre des schönen.) - 
'l'reschow's Nachfolger als Professor der Philosophic in Kopenhagen war Frederik (hristian Sibbern (1785-1872). Er wurde in seiner Jugend von der Romantik und ihrer Philosophie stark ergriffen. In ihnen fand er Nahrung für Her\% und Geist. El war eine dichterische Natur, und hat für die Gährung seiner Jugend in rlen "Briefen Gabrielis" " einen poetischen Ausdruck gesucht. Seine Lehre ist eine der gestïndesten Formen, in welchen die deutsche Philosophie auf das dänische Geistesleben Einfluss gewonnen-hat. Sein Sinn für das Konkrete, sein Beobachtungsinteresse und seine warme Sympathie für das individuelle Leben bewirkten, dass er kein Anhänger des abstrakten und apriorischen Jeducirens und Konstruirens werden konnte. Nach seiner Ueberzcugung musste die Philosophie immer von einem gegebenen Inhalt ausgehen, und erst durch die Bearbeitung dieses Inhalts konnten die höchsten Ideen gewonnen werden. Die Philosophie muss explikativ sein, che sie spekulativ werden kann; und die spekulative Erkenntniss muss selbst wieder durch beständige "Konferen\%" mit dem wirklich Gegebenen bestätigt werden. Wenn die Ideen, die uns die Welt verständlich machen, sich uns bei unserer Bearbeitung der gegebenen Erfahrungen kundgeben, so ist dies nach Sibbern (welcher hier seiner Erkenntnisslehre einen metaphysischen Hintergrund gibt) dadurch möglich, dass wir selbst Glieder des Weltzusammenhangs sind, und dass das innerste Wesen der Welt sich auch in uns regt. Dass aber eine absolute Erkenntniss nicht erreicht werden kann, ist in der sporadischen Entwickelungsweise der Welt begründet. In grosser Mannigfaltigkeit, in vielen einzelnen Erscheinungen auf einmal erscheint uns die Welt, und es ist eine unendliche Aufgabe, das gemeinsame Centrum der verschiedenen Entwickelungsreihen $z u$ bestimmen. (Vergl. die Schrilt von Sibbern „über den Begriff, die Natur und das Wesen der Philosophie" 1843.)

Seine allgemeine Weltanschauung hat Sibbern besonders in seiner "Spekulativen Kosmologie" (1846) dargelegt. Alles Entstehen und alles Werden wird durch wwei Faktoren möglich: der eine ist die in Allem wirkende Ordnung, der andere ist etwas bestimmt Gegebenes, welches mit sich führt, dass in jedem einzelnen Falle jener 
Orduung gemäss eben diese bestimmte Erscheinung und keine andere entsteht. Es gibt immer verschiedene Ausgangspunkte, aus welchen die Processe der Natur ihren.Lauf nehmen. Diese Ausgaingspunkte stehen in gegenseitiger Wechselwirkung, so dass sie nicht nur Aktionspunkte, șondern auch Reaktionspunkte sind. Daher erscheint uns die Natur als ein grosser Wechselwirkungsprocess, eine grosse „Debatte, ein Kampf von allem gegen alles“, und erst durch diese grosse Weltdebatte entfaltet das Dasein seine ganze Fülle. - Sibbern tritt durch diese Auffassung in bestimmtern Gegensatz 20 Hegel und der deutschen Schule, denen er vorwirft, dass sie keine wirklich historische Auffassung des Daseins begründen können. Historische Entwickelung setzt gesonderte Ausgangspunkte in gegenseitiger Wechselwirkung voraus. Die Entwickelung geht darauf aus, Harmonie zwischen den sporadisch eingeleiteten Processen, Ordnung in das Chaos zu bringen. Beim Krystallisationsprocesse z. B. (wie wenn das Wasser friert) entstehen die Nadeln an vielen verschiedenen Punkten, aber schliessen sich nach und nach zusammen und bilden einen kompakten Zusammenhang. Einen ähnlichen Verlauf finden wir bei der Entwickelung des: Foetus, bei der Bildung eines Staates. Und wenn wir zum Trsprunge der Erde und des ganzen Planetensystemes zurückgehen, muss jede Partikel des Urnebels Ausgangspunkt für Anziehung und Abstossung gewesen sein; durch die so hervorgebrachten unendlich mannigfaltigen Wechselwirkungen entstand die jetrige Form der Himmelskörper. Innerhalb dieses grossen Entwickelungsprocesses ist das Entstehen des Menschen aus den langen Reihen früherer organischer Vorfahren eine einzelne Episode.

Trot: der sporadischen Ausgangspunkte vollzieht sich nach Sibbern ein kontinuirlicher Fortgang zu grösserer Harmonie und höheren Lebensformen. Aber wie der Fuetus auf gewissen Stadien seiner Entwickelung dem unkundigen Beobachter eine Missgestalt zu sein scheint, kann auch bisweilen die Entwickelung der Welt in eine ganz andere Richtung als die nach grösserer Vollkommenheit zu gehen scheinen. J)ies sind doch nur Krümmungen des Weges, welche dic immer wachsende Harmonic zwischen dem Einheitsgrunde des Jaseins und den mannigfaltigen Ausgangspunkten nicht aus- 
schlieswen. -- Dio Weltentwickelung ist ewig, weil eine unendliche luhaltsfiille durch sie realisirt werden soll. - Die Welt ist als eine urosic Indivilualitiat \%u betrachten, deren Centrum die Gottheit ist. Die Mannigfaltigkeit der Elemente (dor sporadischen Ausgangspunkte) ist die „Weltseite" des Daseins; das Einheitsprincip, welches sich in der Weltordnung kundgiebt, ist die "Gottesseite" des Daseins. Obgleich Sibbern sich so dem Pantheismus nähert, betrachtet er doch den innersten Grund des I)aseins als ein persönliches Wesen.

W:̈̈hrend Sibbern in seiner Jugend der Kirchenlehre sehr nahe stand und gegen den Rationalismus kümpfte, konnte er der immer mehr zugespitzten Orthodoxie nicht folgen. Es bildete sich bei ihm ein freier religiöser Standpunkt aus, den er in seinen späteren Schriften (besonders in Universitätsprogrammen aus den Jahren 1846-1849) ausgesprochen hat. Er betonte jetzt die rein humane Seite des Christenthums und wandte seine Theorie der sporadischen Entwickelung anch auf das religiöse Leben an: dieses entwickelt sich durch Wechselwirkung verschiedener Richtungen und Persönlichkeiten und ist nur wahr und gesund, wenn jedes Individuum nur dasjenige sucht, was ihm zur Förderung seines geistigen Wohles dienen kann. Gegen den Versuch der Kirche alle religiöse Vorstellungen nach einem Muster'zu formen tritt Sibbern sehr bestimmt auf. Sein Standpunkt ist hier Subjektivismus, aber durch den festen Glauben an das in allen Subjekten sich regende universelle Leben begründet. -

Von einzelnen Disciplinen hat Sibbern sich besonders mit der Psychologie beschäftigt. Er brachte hierzu in mehrfacher Richtung glückliche Voraussetzungen mit: einen frischen und lebendigen Natursinn, gutes Beobachtungsvermögen, besonders für die Erscheinungen des Gefühls- und Trieblebens, und nicht geringe Einsichten in Naturwissenschaft und Physiologie. Seine psychologischen Arbeiten (deren älteste 1819 erschien) leiden teils unter dem berrschenden teleologischen Vitalismus, teils - besonders in den späteren Ausgaben - unter einem Hange zum Rubriciren und Katalogisiren, welcher zu seiner grossen und warmen Sympathie für die seelischen Lebensïusserungen in einem eigentïmlichen Gegensatz steht. Die besten Abschnitte seiner psychologischen Werke 
sind diejenigen, welche das Gefühlsleben behandeln. Besonders die Bedeutung der Kontrast- und Mischungsverhältnisse auf diesem Gebiete hat er in grossen Zügen klar hervorgehoben. - In seinem Buche „Om Forholdet mellun Sjael og Legeme“ (1849) [Ueber das Verhältniss zwischen Seele und Körper] erklärt er sich gegen jede dualistische Auffassung und legt die Theorie zu Grunde, dass das Leben als Bewusstseinsleben und als materielles Leben nur ein Leben ist. „Die körperliche Wirksamkeit und die Geisteswirksamkeit sind wesentlich zusammenhängende koordinirte Wirkungen einer gemeinsamen, gleichzeitig in beiden wirkenden Ursache." Er weist die spiritualistische Lehre von der Seele als einer Substanz oder eines für sich bestehenden Wesens entschieden ab: "Seele ist nur seelisches Leben." - Wie bei Treschow hat auch bei Sibbern die Identitïtshypothese noch nicht jene Klarheit und Nothwendigkeit, welche zuerst Fechner ihr gegeben hat, indem er sie nur als nothwendige Konsequenz des Gesetzes der physischen Energie darstellte. -

Sibbern hatte ein grosses Interesse für alle Seiten des Lebens. Nicht nur an der religiösen, auch an der politischen Debatte nahm er eifrigen Anteil. In allen Stadien unserer inneren politischen Entwickelung ron 1830-1870 hat er durch Brochuren und Abhandhandlungen seine oft sehr gründlichen und originellen Beiträge zu den brennenden Fragen gegeben. Er ging aber immer seinen eigenen Weg, und seine unbeholfene Darstellungsart nebst seinen vielen Idiosynkrasien hinderten ihn hier - wie auch auf anderen Gebieten - daran, einen solchen Einfluss zu üben, wie seine Ideen und sein warmes Herz es an und für sich verdienten. - In seinen let»ten Jahren beschäftigte er sich viel mit der socialen Frage, welche er schon 1849 als weit wichtiger als die konstitutionelle bezeichnete und schrieb u. a. eine Utopie von einem kommunistischen Zukunftsstate, die er in der Form von „Mitteilungen aus einer Schrift aus dem Jahre 2135" herausgab. -

Alles in Allem eine der originellsten Persönlichkeiten, welche das dänische Geistesleben in diesem Jahrhundert aufzuzeigen hat. Lnter den öfientlichen Diskussionen, an welchen Sibbern Teil nahim, war auch der interessante Streit, welcher in den Jahren 
1824-1825 in unserer Literatur uiber die Freiheit des menschlichen Willens geführt wurde. Er begann mit einer Meinungsverschiedenheit zwischen Acrzten und Juristen darüber, ob es - wie diese hehaupteten - eine scharfe Grenze zwischen Wahnsinn und normaler menschlicher Vernunft gäbe, oder ob - wie jene behaupteten - Uebergäinge und Zwischengrade vorhanden seien. In einer scharfsinnigen und interessanten Schrift „Om Afsindighed und Tilregnelse, et Bidrag til Psykologien og Retslaeren" (1824) [Ueber Wahnsinn und Zurechnung, ein Beitrag zur Psychologie und Rechtslehre] unternahm F. G. Howitz, Professor der Medicin an der Universität, eine tiefergehende Untersuchung der Begriffe von Freiheit und Zurechnung. In philosophischer Hinsicht ist er am meisten von Spinoza und Hume beeinflusst. Er fordert eine rein psychologische und physiologische Betrachtung des menschlichen Willenslebens und sucht darzutun, dass eine solche sehr wol mit einer ethischen Betrachtung vereinbar ist. Howitz ist ein klarer und geschmackvoller Autor, dessen Standpunkt dem in der neueren Psychologie und Ethik herrschenden entschieden verwandt ist. In seiner eigenen Zeit stand er einsam. Eine Reihe der ersten Namen der dänischen Literatur traten gegen ihn auf. A. S. Oersted (Dänemarks grösster Jurist) und J. P. Mynster (später Bischof in Kopenhagen) bekämpften ihn . wesentlich vom Standpunkte der gangbaren moralischen Vorstellungen aus, während Sibbern und J. L. Heiberg eine höhere Einheit ron Freiheit und Notwendigkeit aufzuzeigen suchten.

\section{- II.}

Der eben genannte Johann Ludwig Heiberg (1791-1860) war der erste Vertreter der Hegel'schen Philosophie in der dänischen Literatur. Sein Name ist bei uns besonders als Dichter und ästhetischer Kritiker sehr geschätzt; aber seine philosophischen Studien hatten einen grossen Einfluss auf seine Entwickelung nach beiden Richtungen. Als Professor der dünischen Literatur in Kiel (18221825) wurde er mit der Hegel'schen Philosophie bekannt und suchte mit Ernst in sie einzudringen. Es fiel ihm aber sehr schwer, und selbst ein Aufenthalt in Berlin, wo er Hegel's persönlichen Umgang ge- 
noss, half ihm noch nicht, den Grundgedanken des Systems zu erfassen. Auf der Rückreise aber hatte er dann seine philosophische Erleuchtung. Er hielt sich einige Zeit in Hamburg auf, immer mit den neuen Gedanken beschäftigt. Eines Tages - erzählt er selbst - „wurde ich plötzlich in einer Weise, welche ich niemals vorher noch spätér erlebt habe, von einer momentanen inneren Anschauung ergriffen, welche wie ein Blitzstrahl mir auf einmal die ganze Region erlenchtete und den mir bis jetzt verborgenen Centralgedanken in mir weckte. Von diesem Augenblicke an war mir das System in seinen grossen Umrissen klar, und ich war vollkommen davon überzeugt, dass ich es in seinem innersten Kern erfasst hatte ... Ich kann wahrheitsgemäss sagen, dass jener wunderbare Augenblick wol die wichtigste Epoche in meinem Leben war." Diese Erleuchtung (über welche die Gegner des Hegelianismus und Heibergs sich oft lustig gemacht haben) war für ihn auch als Dichter fruchtbar, und seine eigene Erklärung hierüber ist wohl geeignet zu zeigen, in welcher Weise er die Hegel'sche Philosophie auffasste. "Ich hätte niemals meine Vaudevillen geschrieben und wäre gar nicht Theaterdichter geworden, wenn ich nicht durch die Hegel'sche Philosophie das Verhältniss des Endlichen zum Unendlichen zu verstehen gelernt und dadurch einen Respekt vor den endlichen Dingen bekommen hätte, welchen ich vorher nicht fühlte, dessen aber der dramatische Dichter unmöglich entbehren kann, und wenn ich nicht durch dieselbe Philosophie die Bedeutung der Begrenzung zu erfassen gelernt hätte. Denn ohne eine solche hätte ich mich weder beschränkt, noch kleine und begrenztere, vorher ron mir verachtete Rahmen zu meiner Darstellung gewählt." Heiberg machte also die neue Einsicht besonders in seiner poetischen Produktion fruchtbar; aber er hat sie auch in interessanten und geistvollen Abhandlungen auf die theoretische Acsthetik (besonders bei lBestimmung der gegenseitigen Verhältnisse der Kunstarten) angewandt. Ausserdem hat er einen „Leitfaden zu Vorlesungen über spekulative Jogik" geschrieben, und in seiner Abhandlung „über die Bedeutung der Philosophie für die jetzige Zeit" suchte er zu zeigen, wie durch die Hegel'sche Philosophie eine freiere und tiefere IT'eltanschauung erreicht wird: "l)as Ideal wird mit der Il'irklichkeit, 
unsere Forderungen werden mit demjenigen, was wir besitzen, unsere "Wünsche mit demjenigen, was erreicht worden ist, versöhnt." In ler von Heiberg herausgegebenen Zeitschrift „Perseus, Journal für die spekulative Idee" hatte diese Philosophie sogar eine kurze Zeit ihr eigenes Organ.

Neben der Aesthetik wurde der Hegelianismus besonders auf die Theologie angewandt. Eine neue Aera schien Vielen durch die Art, in welcher Hegel Glauben und Wissen versöhnt hatte, eingeleitet zu. sein. W'as auf dem Gebiete des Glaubens in der anschaulichen, konkreten Form der Vorstellung oder der Phantasie hervortritt, sollte dasselbe sein, was für den philosophischen Denker als abstrakter Begriff hervortritt; der Unterschied sollte nur formal sein. "Der Gedanke stieg" - wie Heiberg in einer Universitätskantate sagt - „in die Höhe hinauf, als er in sich selbst niederstieg." Unter diesen grossen Verheissungen ergriff die studirende Jugend eine spekulative Raserei. Man führte immer Hegel'sche Formeln im Munde und verachtete diejenigen, welche auf dem Standpunkte der "Unmittelbarkeit“ standen, ohne sich zum „Begriffe“ erhoben zu haben. Alles konnte man auflösen und zu einer "höheren Einheit" verbinden. - Vorläufig war es die sogenannte "rechte" (orthodoxe; konservative) Seite der Hegel'schen Schule, welche Anhänger gewann. Als Gegner des Hegelianismus traten Mynster und Sibbern auf. Paul Möller ( $†$ 1838), Sibberns Kollege an der Universität, auch als Dichter bekannt, hatte sich eine Zeitlang dem Hegelianismus genähert, aber entfernte sich später von ihm und nahm einen, dem jüngeren Fichte am nächsten verwandten Standpunkt ein. Er hat sich zumeist mit der Geschichte der Philosophie beschäftigt. H. Martensen, der Theologe, Mynster's Nachfolger als Bischof in Kopenhagen, stand Heiberg nahe und war Mitarbeiter am „Perseus"; aber er fühlte sich durch Hegel's Versuch einer autoritätslosen und voraussetzungslosen Philosophie abgestossen, und wollte, dass die Philosophie statt eines "autonomen" einen "theonomen" Ausgangspunkt nehmen solle. Von der entgegengesetzten Seite wurde der Versöhnungsversuch durch Anhïnger der linken Seite des Hegelianismus, besonders A. F. Beck und H. Bröchner, bedroht. Doch der bedeutungsvollste 
Widerstand gegen diese ganze Philosophie kam von dem Manne, welcher Dänemarks grösstes Denkergenie in diesem Jahrhundert ist, Sören Kierkegaard $\left.(1813-1855)^{1}\right)$.

Es ist für Kierkegaard charakteristisch, dass er sich in seiner Jugend viel mit Sokrates beschäftigt hat. Seine Doktordisputation: „Om Begrebch Ironi med saerligt Hensyn til Sokrates" (1841) [Ueber den Begriff der Ironie mit besonderer Rücksicht auf Sokrates] gibt eine sehr geistvolle, obgleich einseitige Charakteristik des Sokrates. Bei der ganzen folgenden literarischen Wirksamkeit Kierkegaards blieb Sokrates sein Vorbild. Er liebte die indirekte und experimentirende Darstellungsart im Gegensatz zu der direkten und dogmatischen, welche von den spekulativen Philosophen und Theologen angewandt wurde. Die Gymnastik der Gedanken war seine Lust, und sein grosses Talent mit Begriffen zu operiren war mit einer ausserordentlichen Sprachkunst, freilich auch mit einer Neigung sowohl mit Begriffen als mit Worten zu spielen, verbunden. Alle diese Talente wandte er im Dienste des religiösen (ilaubens an. Er betrachtete es als seine Aufgabe, das religiöse Problem wieder in seiner strengen christlichen Form $\mathrm{zu}$ stellen, nachdem die Versöhnung des Christenthums mit der Welt im Staatskirchentume („dem officiellen Christentume") und die Versöhnung von Glauben und Wissen in der spekulativen Philosophie die Einsicht verdrängt hatte, dass es sich hier nur um das persönliche Verhältniss des Einzelnen der absoluten Forderung gegenïber handelt. Für diese Aufgabe wirkte er durch seine ganze Produktion, durch seine ästhetischen und philosophischeu sowol wie durch seine religiösen Schriften, auch daun, als er als einsamer Denker und Schriftsteller seine nur von Wenigen recht verstandenen Werke schrieb, da er - in seiner letzten Lebenszeit - als leidenschal'tlicher Polemiker und Agitator einen gewaltigen Kampl gegen die Staatskirche begann.

1) Celser die Persïnlichkeit, das Leben und Wirken dieses merkwürdigen Jaunes vgl. die von $\mathrm{sehr}$ verschiedenen Standpunkten geschriebenen Karakteristiken von Georg Brandes („S̈̈ren Kierkegaard, eine kritische Darstellung") und A. Bärthold (,S. K., eine Autor-Existen\% eigener $\Lambda$ t $^{\text {" }}$; "Noten zu S. K.'s Lebensgeschichte"; . Die Bedeutung der ästhetischen Schriften S. K. ${ }^{\star}{ }^{\star}$ ).

Archiv f. rimschioher d. Philosophic. II. 
In philosophischer Hinsicht ist die bedeutendste Schrift Kierkegaard's "I'videnskabelig Efterskrift" (1846) [Unwissenschaftliche Nachschrift]. Er bekämpft hier den spekulativen Idealismus. Seine Hauptsiitze sind: Ein logisches System kann es geben, aber cin System der Wirklichkeit kann es - für uns Menschen nicht geben. Und selbst was das logische System, welches die allgemeinen Kategorien des Daseins darstellt, betrifft, muss zwischen den rein apriorischen und den von der Wirklichkeit abstrahirten Begriffen unterschieden werden. Das logische System soll keine Mystifikation sein, in welcher der Inhalt der Wirklichkeit durch Subreption entwickelt wird. Man muss sich ferner darüber klar sein, dass der Anfang des Systems nur durch Reflexion und Abstraktion gemacht wird, so dass von einem absoluten Anfang keine Rede sein kann. Endlich muss scharf zwischen dem empirischen Leben und der dem Iogischen Systeme zu Grunde liegenden reinen oder abstrakten Subjektivität unterschieden werden. - Ein System der Wirklichkeit kann es für uns nicht geben, weil unsere Existenz in die Zeit fällt. Existiren ist Werden. System bedentet Abgeschlossenheit, Totalität, aber so lange wir existiren, streben wir und können nicht unser und das ganze Dasein zusammenfassen. Nur für Gott bildet die Wirklichkeit ein System, weil er über das Werden erhaben ist. Ein jedes System muss pantheistisch sein und nicht nur den Unterschied zwischen Gut und Böse aufheben, sondern auch den Begriff der Existenz in phantastischer Art verflüchtigen. Eben die Abgeschlossenheit macht das System pantheistisch. [Kierkegaard sieht nicht die naheliegende eigentümliche Konsequenz, dass Gott also Pantheist sein muss!] Es hilft nichts, dass man einen Paragraphen einschiebt, in welchem man sagt, dass man die Existenz urgire. Wenn man wirklich die Existenz urgirt, muss dies sich darin zeigen, dass man kein System (der Wirklichkeit) aufstellt. - Ein solches System würde keine Ethik aufstellen können. Das ethische Willensleben setzt die Realität der Aufgabe und der Wahl voraus, und verschwindet in seiner Bedeutung, wenn man die Welt sub specie aeterni sieht.

Für die in der Zeit existirende Subjektivität kann die Wahrheit nur im Glauben erfasst werden; eine objektive Gewissheit ist 
unmöglich. Nur durch leidenschaftliche Anschliessung kann die Wahrheit unter dem Wechsel der Wirklichkeit festgehalten werden. Die Wahrheit ist das Ewige, aber wir sind in der Zeit. Aber der Glaube erreicht seine höchste Potenz, wenn sein Gegenstand nicht nur die ewrige Einheit ist, die wir nur wegen des Wechsels der Wirklichkeit nicht objektiv̄ begründen können, sondern wenn die ewige Wahrheit selbst in der Zeit entsteht, wie Gott im Christentume in einem bestimmten Zeitpunkte Mensch wird. Der christsiche Glaube ist der Glaube an das Paradoxe, und nur die Verzweiflung des Sündenberwusstseins macht es möglich, den Sprung von jenem sokratischen Glauben zu dieser höchsten Potenz des Glaubens zu machen.

Kierkegaard accentuirt mit solcher Energie die Bedeutung der einzelnen Subjektivität, dass er alle Kontinuität zwischen den Individuen und der übrigen Welt aufhebt. Er polemisirt gegen die Lehre von den „zurückgelegten Stadien“. Geistesentwickelung sagt er - ist Selbstentwickelung; jedes folgende Individuum muss von vorn anfangen. Das menschliche Individuum verhält sich nicht zur Entwickelung des Menschengeistes, wie das Thierexemplar sich zur Thierart verhält. - Man kann hieraus urteilen, wie Kierkegaard sich zur modernen Entwickelungslehre gestellt haben würde. Er würde deren Bedeutung nicht anerkannt haben, wie er im Ganzen eigentlich gegen eine jede zusammenhängende und rationelle (physiologische, psychologische und historische) Auffassung des Menschenlebens protestirt. Anderseits hat er Recht darin, dass man den Unterschied zwischen autogenetischer und phylogenetischer Entwickelung nicht verwischen darf; es ist dies - wie früher in der spekulativen Philosophie, so jetzt in der spekulativen Biologie - oft geschehen. -

Kierkegaard's Kritik der spekulativen Philosophie bezeichnet bei uns einen Wendepunkt und bahnt der Erneuerung der kritischen Philosophie den Weg. - Seine strenge Betonung des Vernunftwidrigen des christlichen Glaubensinhalts zog eine scharfe Grenzlinie zwischen positiv-religiösem Glauben und humaner Lebensanschauung, eine Grenzenlinie, welche die Philosophie der Romantik verwischt hatte. Er wirkte als Stromteiler, führte die einen $z u$ 
innigerer Vertiefung in das Glaubensleben, aber machte es für die anderen klar, dass ihre Juebensanschauung eigentlich gar nicht auf dem Boden des Christentums beruhe. Er steht in unserer Literatur als eine sokratische Gestalt da, welche auf viele Menschen, mögen sie auch sehr verschiedene Standpunkte einnehmen, einen anregenden und erlösenden Einfluss geübt hat. -

Ein Anhänger der Hegel'schen Religionsphilosophie war in seinen ersten Arbeiten Rasmus Nielsen (f. 1809, von 1841 an Professor der Philosophie an der Universität, gest. 1884). Eine Aenderung seiner Ansichten wurde durch S. Kierkegaard's Schriften hervorgerufen und er brachte sie zuerst in „Evangelietroen og den moderne Bevidsthel" (1849) [Der Evangelienglaube und das moderne Bewusstsein] und dann in einem scharfen Angriffe auf die Dogmatik Martensen's zum Ausdruck. Nielsen unterschied von dieser Zeit an zwischen Glauben und Wissen als zwei absolut verschiedenen Principien. Der Glaube ist die Sache des Lebens, der Existenz und hat im Wollen seine Wurzel; das Wissen ist die Sache der objektiven Theorie. Der Wille selbst ist antirational, unbegreifbar; denn die Theorie begreift nur eine Freiheit, die mit innerer Notwendigkeit eins ist. Je energischer der Wille entwickelt ist, desto schärfer ist der Gegensatz des Willenslebens gegen die Theorie, und der Mensch kann nicht unbedingt wollen, ohne die Gottheit als unbedingt allmächtigen Willen zu postuliren. Der Glaube ist ein Glaube an das Wunder. Aber eben darum hat das Wunder auch nur Realität für den Glauben, hat nur eine geistige Wirklichkeit. Darum ist keine Theologie als Wissenschaft möglich. Nielseu polemisirt gegen die Theologie, aber behauptet den Glauben. Die Kritik der Theologie geht seiner Auffassung nach zu weit, wenn sie den religiösen Glauben selbst angreift. Eben weil Glaube und Wissen absolut verschiedene Principien sind, können sie beide festgehalten werden. Der Glaube ist die Sache der persönlichen Lebensanschauung und muss daher die höchste Stelle einnehmen; die Wissenschaft ist nur eine relative Aufgabe und kann dem Existirenden in seinem persönlichen Leben nicht helfen. - In seiner "Religionsphilosophie" (1869) hat Nielsen diese Gedanken ausführlich entwickelt. 
Der Bruch mit der spekulativen Religionsphilosophie führte Nielsen zu einer neuen Prüfung des Verhältnisses zwischen Spekulation und Erfahrung und dadurch zu einem eifrigen Studium von Mathematik und Naturwissenschaft. Doch gab er niemals die Hegel'sche Methode auf. Er hoffte nur, sie auf einer solideren Grundlage durchführen zu können. In „Grundideernes Logik“ (1864-1866) und "Natur og Aand". (1873) [Natur und Geist] findet man seine allgemeinen philosophischen Anschauungen. Es ist die Aufgabe der Philosophie den Inhalt der Fachwissenschaften zu assimiliren und die Begriffe ron ihrer ursprünglichen empirischen Begrenzung zu befreien, damit das Bewusstsein den so erworbenen Inhalt zu einer Totalität mit innerem Zusammenhang formen kann. Hegel hatte in unklarer Weise das Subjektive und das Objektive, den Begriff und die Existenz identificirt. Im Gegensatz $z u$ ilim behauptet Nielsen den Unterschied zwischen der wissenden Subjektivität und der objektiven Realität und stellt darum - statt einer „Logik der Idee“ - eine "Logik der Grundideen“ auf, indem die Idee des Wissens und die Idee der Macht ihren Dualismus in der Idee der Wahrheit, welche mit der absoluten (ontologischen) Subjektivität eins ist, überwinden. Hegel's Grundfehler war - nach Nielsen dass das absolute Wissen bei ihm wol einen Inhalt, aber keinen Gegensatz hat. Die absolute Subjektivität muss nicht nur das absolute Wissen, sondern auch die absolute Macht sein; nur dann können die logischen Kategorien Wirklichkeits-Kategorien werden.

Die Philosophie führt also zu einem Theismus; aber dieser ist zu abstrakt, um Grundlage der praktischen Lebensanschauung zu sein; darum muss man - wenn das persönliche Leben nicht erschlaffen soll - rom Wissen zum Glauben übergehen. - Esist für Nielsen eigentümlich, dass er die Gegensätze so stark hervorhebt. Dies hängt gewiss damit zusammen, dass bei ihm die Phantasie beinahe der Reflexion ebenbürtig war, wie er denn überhaupt ein lebhafter und energischer Redner war, der es besonders liebte, sich in Antithesen zu heweren. Grossen Einfluss hat er durch seine zündenden Vorträge auf die akademische Jugend und auf weitere Kreise ausgeübt. Jie Gegensätze stellen ja eben die Probleme und fordern die Denkliraft heraus. - 
Die Lehre Nielseu's vom Glauben und Wissen gab in den Jahren 1865-1868 zu einer Polomik Anlass, in welcher eine Reihe älterer und jüngerer Autoren auftraten. Die grösste Aufmerksamkeit erregte von der einen Seite eine Schrift von Martensen, von der anderen Georg Brandes durch seine Schrift ngegen den Dualismus unserer neuesten Philosophie" (1866). Aber die gründlichste Untersuchung der ganzen Frage gab H. Bröchner's Schrift „Problemet om Tro og Viden“ (1868) [„Das Problem vom Glauben und Wissen"].

Hans Bröchner (geb. 1820, von 1857 Professor der Philosophie an der Universität, gest. 1875) war von der Theologie zur Philosophie übergegangen, hatte Strauss's "Glaubenslehre" übersetzt und später (1857) eine gründliches Studium und tiefe Sympathie bekundende Abhandlung über Spinoza geschrieben. Auch Bröchner gehörte der Hegel'schen Schule an und arbeitete sich, eben so wenig wie Nielsen, niemals aus ihrem Gedankengang und ihrer Methode heraus. Er suchte doch zu zeigen, dass dieser Gedankengang so entwickelt werden könnte, dass die Erfahrung und die reale Wirklichkeit ihr Recht bekämen. Am meisten beschäftigte er sich mit der Geschichte der Philosophie. Die Frucht dieser Studien hat er teils in der geistreichen Schrift: „Bidrag til Opfattelsen af Philosophiens historiske Udvikling" (1869) [Beiträge zur Auffassung der historischen Entwickelung der Philosophie], teils in seinem Handbuch der Geschichte der Philosophie (1873-1874) niedergelegt. Der oben genannten Streitschrift, in welcher er sich sowol gegen Martensen ("die philosophirende Theologie“), als gegen Kierkegaard und Nielsen („den antitheologischen religiösen Standpunkt") wendet, gab er ein positives Supplement in der Schrift: "Det Religiöse i dets Enhed med det Humane" (1869) [Das Religiöse in seiner Einheit mit dem Humanen]. Nach Bröchner ist die wahre Religiösität nicht etwas von dem wahren Erkennen und dem wahren (ethischen) Handeln Verschiedenes. Sie ist die Versöhnung und die Ruhe, welche der Menschengeist findet, wenn er sich aus dem unmittelbaren sinnlichen Leben, wo er von streitenden Kräften geteilt wird, und wo der Zweifel an der Wahrheit der Erkenntniss und der Gültigkeit der Handlung entstehen kann, zu seinem Innern, 
dem Grunde seines Wesens zurückzieht. Hier findet er die innere Quelle seiner Kräfte, und hier sieht er seinen Geist in seinem Ursprunge aus dem allgemeinen Princip des Daseins. Diese Einsicht kann wegen der Begrenzung des menschlichen Wesens nicht vollständig bewiesen werden; sie ist ein rationeller Glaube, welcher aus dem Wesen des Menschen entspringt. Die Erkenntnișs hat hier eine regulirende Macht; sie kann wol einem Glaubenspostulate gegenïber ihr non liquet aussprechen, behält aber immer ihr veto. Sonst würde jedes Kriterium zwischen wahrem und falschem Glauben mangeln. - Das Princip des Daseins muss als ideal-reales gedacht werden, so dass es die reale Möglichkeit der Materie in sich fasst. Es ist eine absolute Subjectivität, die Quelle des persönlichen Lebens, aber selbst über alles persönliche Dasein erhaben.

Diese seine Auffassung, welche er durch viele tiefgehende psychologische Entwickelungen (so namentlich über das Problem des Bösen) begründet, stellt Bröchner in Gegensatz teils $\mathrm{zu}$ solchen Ansichten, welche das Religiöse entweder in metaphysische Abstraktionen oder psychologische Illusionen auflösen, teils zu den positiven Religionen, welche das wahrhaft Religiöse verendlichen, indem sie es in äusserliche und endliche Formen kleiden. - Bröchner hat - obgleich er sowol durch seine abstrakte und schwer zugängliche Darstellungsart als durch die Richtung seiner Ideen in seiner Zeit sehr einsam stand - durch die Idealität und Energie seiner Persönlichkeit auf nicht wenige jüngere Männer gewirkt, die er in eine freie und humane Lebensanschauung eingefïhrt hat. -

\section{III.}

Nur eine kurze Uebersicht über die Literatur der letzten Decennien soll noch hinzugefügt werden. Bis 1870 war die spekulative Philosophie die allein herrschende in dem öffentlichen philosophischen Lnterricht. Die Reaktion gegen sie ist dem Einflusse teils der englisch-französischen Philosophie, teils den lachwissenschaften zuzuschreiben. Die erste Arbeit, in welcher eine solche Reaktion hervortritt, ist die Doktorabhandlung des bekannten Aesthetikers Georg Brandes (geb. 1842) über Taine's Aesthetik 
(1870). - In den bolgenden Jahren gats der Verfasser dieser Uoljersicht (gch. 18+3), seit 1883 Professor an der Universität, Arbeiton ïber die nachliegelsche Philosophie in Deutschland (1872) und über die englische Philosophic unserer Zeit (1874) heraus. Innerhalb der deutschen Philosophie wurde hier namentlich Lotze's Becloutung hervorgehoben, innerhalb der englischen auf Stuart Mill und namentlich auf Herbert Spencer hingewiesen. In einer Abhandlung über "die Grundlage der humanen Ethik" (1876) (in deutscher Uebersetzung 1880) versuchte ich eine rein psychologischhistorische Grundlegung der Ethik. In den folgenden Jahren beschäftigten mich namentlich psychologische Studien, deren Frucht die im Jahre 1882 erschienene "Psychologie im Umriss“ war. (Zweite Auflage 1885. Deutsche Uebersetzung 1887). Ich habe hier eine rein empirische Darstellung der psychologischen Hauptfragen versucht, mit grösstmöglicher Benutzung der Physiologie und der Experimentalpsychologie. Obgleich ich der englischen Schule sehr viel verdanke, habe ich doch verschiedene ihrer Resultate zu berichtigen versucht, und in philosophischer Rücksicht führen meine Untersuchungen mich dazu, die Grundgedanken des Kriticismus als Ergebnisse der psychologischen Analysen und Hypothesen zu behaupten. In meiner "Ethik" (1887) (Deutsche Uebersetzung 1888) habe ich versucht; teils eine Darstellung der ethischen Principien zu geben, teils diese Principien durch eine Untersuchung der wichtigsten Lebensverhältnisse zu prüfen und anzuwenden. Sophus Heegaard (geb. 1835, seit 1875.Professor der Philosophie, gest. 1884) bildete seine philosophischen Anșichten namentlich unter dem Einfluss eines eifrigen Studiums von Kant und Lotze, später auch von Mill und Spencer aus. Als Schriftsteller ist er am meisten durch seine populäre Schrift über „Intoleranz" (1878) bekannt, in welcher er das Recht der einzelnen Individuen, ihre religiösen Anschauungen auf eine mit ihrer Persönlichkeit stimmende Weise zu formen, behauptet. Er steht jedem Versuche einer wissenschaftlichen Entscheidung religiöser Fragen skeptisch gegenüber, aber betont sehr stark, dass die Möglichkeit, der Glaube (besonders der Unsterblichkeitsglaube) könnte Recht haben, von grosser Bedeutung für die Lebensführung ist. ("Wir leben von Möglichkeited.“) 
Später hat er ein Handbuch der Erziehungslehre geschrieben. Kristian Kroman (geb. 1846, seit 1884 Professor der Universität) gab schon in seiner Doktorabhandlung „über die Lehre der exakten Wissenschaft von der Existenz der Seele" (1877) Andeutungen über den Standpunkt, welcher in seinem Hauptwerke: "Vor Naturerkendelse" (1883) (Deutsche Uebersetzung 1883) hervortritt. Er nimmt allen spekulativen Anschauungen und dogmatischen Behauptungen gegenüber einen skeptischen Standpunkt ein. Die Principien unserer Erkenntniss sind ihm nur Postulate, welche weder deduktiv, noch induktiv begründet werden können. Die erkenntnisstheoretischen Fragen werden mit gründlicher mathematischer und naturwissenschaftlicher Einsicht auf das Gebiet der Fachwissenschaften hinüber .verfolgt. In seinen allgemeinen philosophischen Ansichten scheint Kroman Lotze am nächsten zu stehen. Ausser einem Kompendium über Logik und Psychologie (1882) (Zweite Auflage 1888) hat er noch „über Ziele und Wege des höheren Schulunterrichts" (1886) geschrieben. Diese letzte Schrift, welche in-lebhafter Form die pädagogische Bedeutung der verschiedenen Lehrfächer diskutirt und sich namentlich gegen das Vorherrschen des Sprachunterrichts wendet, hat grosse Aufmerksamkeit erregt und den Streit um die Ordnung der Schule belebt. Claudius Wilckens, seit 1884 Docent der Philosophie und der Sociologie an der Universität, hat in seiner Doktorabhandlung „über das Erkenntnissproblem" (1875) einen durch den Einfluss der Beneke'schen Philosophie modificirten Kriticismus aufgestellt. In seiner "Sociologie" (1881) gibt er eine auf fleissiges Studium gebaute Darstellung dieser Disciplin, und so eben ist (1888) von seiner Hand eine "A esthetik" erschienen. - Auf sociologischem Gebiete arbeitet auch Jr. C. N. Starcke, dessen Buch „Die primitive Familie“ (1888 in Jeipzig erschienen) eine eingehende Lntersuchung über die primitiven Familienverhältnisse, welche ja in den letzten Jahren mehrere gewagte Theorien veranlasst haben, licfert. Früher hat Starcke iiber Ludwig Fenerbach (1883) (Jeutsche Cebersctzung 1885) geschrieben. - In J)r. Alfred Lehmann haben wir einen selbstïndigen Arbeiter auf dem Gebiete der experimentellen Psychologie. Ausser seiner Doktorabhandlung „über die elementare Aesthetik 
der Farben" (1884) hat er in Wundt's "Studien" verschiedene Abhandlungen veröffentlicht. -

Als gemeinsam für die jüngere philosophirende Generation in Jänemark darf ich gewiss die Ueberzeugung angeben, dass es in der Philosophie jetyt vor allem darum, zu thun ist, durch Untersuchungen auf dem Gebiete der Erkenntnisslehre, der Psychologie, der Ethik und der Socialwissenschaft eine solche Grundlage herbeizuschaffen, durch welche die grossen philosophischen Probleme beleuchtet werden können. Aber trotz aller unserer Kritik und alles unseren Realismus müssen wir einräumen, dass der Grundgedanke des Idealismus die letzte Voraussetzung ist, zu welcher die Reflexion uns zurückführt, selbst wenn wir diesen Gedanken nicht in so dogmatischer Weise aussprechen oder so grosse Hoffnungen auf seine wissenschaftliche Durchführung setzen können, wie es bei unseren Vorgängern der Fall war. - 